\title{
Altered frontal cortical volume and decision making in adolescent cannabis users
}

\author{
John C. Churchwell ${ }^{1}$, Melissa Lopez-Larson ${ }^{1,2}$ and Deborah A. Yurgelun-Todd ${ }^{1,2 *}$ \\ The Brain Institute, University of Utah, Salt Lake City, UT, USA \\ 2 Department of Psychiatry, University of Utah School of Medicine, Salt Lake City, UT, USA
}

Edited by:

Reinout W. Wiers, University of

Amsterdam, Netherlands

Reviewed by:

Susan F. Tapert, University of California

at San Diego, USA

Dick Veltman, Free University,

Netherlands

*Correspondence:

Deborah A. Yurgelun-Todd, The Brain

Institute, University of Utah, 383

Colorow Drive, Salt Lake City, UT

84108, USA.

e-mail: deborah.yurgelun-todd@hsc. utah.edu
Anticipating future outcomes is central to decision making and a failure to consider long-term consequences may lead to impulsive choices. Adolescence is a vulnerable period during which underdeveloped prefrontal cortical systems may contribute to poor judgment, impulsive choices, and substance abuse. Conversely, substance abuse during this period may alter neural systems involved in decision making and lead to greater impulsivity. Although a broad neural network which supports decision making undergoes extensive change during adolescent development, one region that may be critical is the medial prefrontal cortex. Altered functional integrity of this region may be specifically related to reward perception, substance abuse, and dependence. In the present investigation, we acquired structural magnetic resonance images (MRI), using a $3 T$ Siemens Trio scanner, from 18 cannabis abusing adolescents (CA; 2 female and 16 male subjects; mean age, 17.7 years; range 16-19 years), and 18 healthy controls (HC; 6 female and 12 male subjects; mean age, 17.2 years; range 16-19 years). In order to measure medial orbital prefrontal cortex (moPFC) morphology related to substance abuse and impulsivity, semiautomated cortical reconstruction and volumetric segmentation of MRIs was performed with FreeSurfer. Impulsivity was evaluated with the Barratt Impulsiveness Scale (BIS). Our results indicate that cannabis abusing adolescents have decreased right moPFC volume compared to controls, $p=0.01, d=0.92, \mathrm{Cl}_{0.95}=0.21,1.59$. Cannabis abusing adolescents also show decreased future orientation, as indexed by the BIS non-planning subscale, when compared to controls, $p=0.01, d=0.89, \mathrm{Cl}_{0.95}=0.23,1.55$. Moreover, total moPFC volume was positively correlated with age of first use $r(18)=0.49, p<0.03$, suggesting that alterations in this region may be related to initiation of cannabis use or that early initiation may lead to reduced moPFC volume.

Keywords: adolescence, cannabis, prefrontal, orbitofrontal, decision making, impulsivity, marijuana, development

\section{INTRODUCTION}

Adolescence is a time during which a variety of factors may converge to increase the likelihood of substance use initiation, abuse, and dependence (Schepis et al., 2008). Adolescents may be particularly susceptible to cannabis use since it is the most commonly abused substance worldwide with North America ranking second for reported utilization (UN, 2008). Adolescence may also be a time of vulnerability to addiction, as it has been reported that early initiation increases the risk for cannabis dependence (Chen et al., 2005). A greater risk for dependence may be associated with an increased sensitivity to the rewarding properties of drugs during this developmental stage (Spear, 2000) and cannabis abuse may disrupt normal neuromaturation and reward sensitivity (Crews et al., 2007). Consistent with this perspective, preclinical models indicate that cannabinoid receptor type $1\left(\mathrm{CB}_{1}\right)$ significantly changes density in prefrontal cortex and other reward related brain regions during adolescence (Ellgren et al., 2008), suggesting a possible interval of neural vulnerability to cannabis exposure.

Preclinical models have provided further evidence that chronic or escalating doses of delta-9-tetrahydrocannabinol produce alterations in prefrontal and nucleus accumbens dendritic morphology 30 days following treatment (Kolb et al., 2006) and this cellular reorganization may be related to disruptions in the function of these structures which are normally involved in reward representation and goal-directed behavior (Robinson and Kolb, 2004; Kalivas and Volkow, 2005). In accordance with this view, animal models have shown that brain reward systems become hypersensitive to drugs of abuse such as heroin and cocaine following adolescent cannabis exposure (Ellgren et al., 2007; Higuera-Matas et al., 2008). Collectively, these studies indicate that the transition from childhood to adulthood involves a critical window during which cannabis use can impact normal remodeling of the prefrontal cortex (Egerton et al., 2006; Crews et al., 2007) and possibly alter the incentive salience of other drugs of abuse (Robinson and Berridge, 1993).

The neurocognitive impact of initiating cannabis use early in life is also of great concern (Medina et al., 2007; Jacobus et al., 2009). Studies employing neuroimaging techniques have shown that prefrontal cortical gray matter undergoes significant morphological change during adolescence and research investigating prefrontal functioning during this period suggests that delayed development of this region may be related to both cannabis use and risk for substance abuse. For example, Medina et al. (2007) demonstrated that adolescent cannabis users who were 
abstinent for nearly a month showed mild impairments across several dimensions of neuropsychological functioning including psychomotor speed, attention, and memory. Cannabis use during adolescence has also been related to altered neural activity in studies using functional magnetic resonance imaging (fMRI) techniques paired with behavioral assays of frontal function. For example, compared to controls, abstinent adolescent cannabis users show decreased functional activity of the dorsolateral prefrontal cortex during performance of a spatial working memory task (Schweinsburg et al., 2008) and, consistent with this observation, adult cannabis users have also been shown to have altered frontal activity in a working memory task (Kanayama et al., 2004). Cannabis users who initiated use before the age of 17 have been shown to have decreased frontal cortical gray matter compared to those who initiated after the age of 17 (Wilson et al., 2000) and early initiation, compared to late initiation, has been associated with poorer performance on neuropsychological tests (Pope et al., 2003). Additionally, a negative correlation between frontal activation and risk for substance use disorder, as indexed by neurobehavioral disinhibition, has also been demonstrated in adolescents (McNamee et al., 2008). These studies suggest that cannabis abusing or at-risk adolescents show altered frontal function and potentially developmentally delayed frontal maturation. Thus, during adolescence, protracted development of frontal cortical systems, in tandem with reduced structural connectivity and peak reward related sensitivity, might contribute to suboptimal decision-making capacities and increased impulsivity (Luna and Sweeney, 2004; Yurgelun-Todd, 2007; Ernst and Fudge, 2009; Somerville and Casey, 2010).

Morphogenesis of the prefrontal cortex has been associated with an enhanced capacity to temporally organize action plans and choices as a means of reaching goals (Fuster, 2002). It has been hypothesized that the orbital and medial regions of the prefrontal cortex are the critical loci for representing goals and computing choice based on reward value (Rolls and Grabenhorst, 2008). In accordance with a role for this region in reward evaluation, planning, and goal-directed action, impaired decision making and impulsivity are characteristic of damage to the medial prefrontal cortex (Bechara et al., 2000; Berlin et al., 2004). Suboptimal decision making, impulsivity, and substance abuse have been related to an inability to reflect on future prospects, a function thought to be mediated by the ventromedial prefrontal cortex (Bechara, 2005). Although impulsivity is a complex construct, trait impulsivity has been associated with several independent dimensions such as decreased attention, motor control, and future orientation, factors indexed by the Barratt Impulsiveness Scale (BIS; Barratt, 1994; Moeller et al., 2001). Studies applying morphometric imaging techniques have demonstrated that alterations in medial orbital prefrontal (moPFC) gray matter volume are correlated with observed and self-reported reported impulsivity in adults and adolescents. For example, a negative correlation between right moPFC gray matter volume and the BIS non-planning subscale was demonstrated in a large community sample including male and female adults (Matsuo et al., 2009) and decreased right moPFC cortical volume was also shown to be associated with increased observed impulsivity in a sample of adolescent males (Boes et al., 2009).
Based on the findings above, we reasoned that adolescent cannabis users may be more impulsive and have altered orbital prefrontal volume in contrast to healthy controls. Accordingly, we acquired MRIs from a community sample of healthy adolescents and adolescents with a history of cannabis abuse to examine whether cannabis abuse might be associated with increased self-reported impulsivity and alterations in orbital prefrontal cortex volume.

\section{MATERIALS AND METHODS SUBJECTS}

Eighteen adolescents with cannabis abuse ( 16 males, 2 females, mean age $=17.7$ years; range $16-19$ years) and 18 healthy controls (12 males, 6 females, mean age $=17.2$ years; range $16-19$ years $)$ were recruited from the Salt Lake City community. The following exclusionary criteria were applied: (1) presence of any current Axis I disorder other than cannabis abuse (for the cannabis group); (2) presence of a serious medical illness, including diabetes; (3) presence of a neurological disorder; (4) history of head trauma resulting in loss of consciousness requiring hospital evaluation; (5) history of ECT treatment; (6) estimated IQ $<85$ (based on measures of verbal fluency and academic history); (7) claustrophobia or metal implanted within the body. Once potential participants were identified as study candidates, cannabis users were instructed to continue use as usual until the study visit. All participants were enrolled in either high school, college, or had recently graduated high school with plans to attend college. All participants also reported average to above average success in academic achievement. We utilized verbal fluency performance as an estimate of IQ. Previous research has found modest to moderate correlations between verbal fluency and estimates of intelligence (for review see Strauss et al., 2006). Cannabis abusing subjects in the present study performed somewhat better on the verbal fluency test $(M=45.8, \mathrm{SD}=9.5)$ than healthy controls $(M=37.5$, $\mathrm{SD}=8.7)$. Neither groups showed clinically significant levels of anxiety as assessed with the Hamilton Anxiety Rating Scale (HAM-A; HC, $M=1.27, \mathrm{SD}=1.93$; CA, $M=2.0, \mathrm{SD}=2.66$ ) or depression as assessed with the Hamilton Depression Rating Scale (HAM-D; HC, $M=0.77, \mathrm{SD}=1.43$; CA, $M=2.11$, SD = 3.07) (Hamilton, 1960, 1969).

\section{PROCEDURE}

The University of Utah School of Medicine institutional review board approved the study and all participants read the informed consent and assented to participate in the study. Parents were required to consent for participants less than 18 years of age. A certificate of confidentiality was also used to protect personal information regarding substance use activities from forced disclosure. All participants were interviewed using a modified version of the Structural Clinical Interview for DSM Disorders (SCID), HAM-A, and HAM-D, to determine lifetime psychiatric history and cannabis use history and abuse/dependence status (Table 1). All cannabis using participants met criterion for cannabis abuse. On the day of the study visit, MRIs and self-report measures of impulsivity were acquired. After acquiring MRIs, subjects were escorted to a separate testing room and administered the BIS version 11. The BIS is a 30 item self-report questionnaire that indexes three independent 
dimensions of impulsivity: non-planning, motor, and attention (Patton et al., 1995). These dimension are related to future versus present orientation, acting without thinking, and attentional vigilance, respectively. Scores on each dimension are summed to obtain an overall impulsivity score. The SCID, HAM-A, and HAM-D were also administered. All subjects also provided a urine sample under the direct observation of a same-gender member of the research group immediately prior to scanning (ARUP Drugs of Abuse Panel 9: ARUP Laboratories, Salt Lake City, UT, USA). An aliquot of the urine underwent standard laboratory urinalysis, which included gas chromatography-mass spectroscopy (GC-MS) to quantify the level of nor-9-carboxy-delta 9-tetrahydrocannabinol (THCCOOH).

\section{MRI ACOUISITION AND IMAGE ANALYSIS}

All acquisitions were performed with a Siemens 3-T Trio magnet using a 12 channel head coil and T-1 weighted 3D MPRAGE sequence: field of view $256 \mathrm{~mm}$, TR $2000 \mathrm{~ms}$, TE $3.38 \mathrm{~ms}$, flip angle $8^{\circ}, 1 \mathrm{~mm}$ slice thickness. High-resolution T1 MPRAGE volumes in DICOM format were anonymized, imported into the FreeSurfer (http://surfer.nmr.mgh.harvard.edu/) image analysis environment, and transformed into the MGZ format recognized by the analysis package. Standard semi-automated workflow procedures employing both surface-based and volume-based pipelines were

Table 1 | Demographic and clinical description of subject sample.

\begin{tabular}{lll}
\hline & $\begin{array}{l}\text { Healthy controls } \\
(\boldsymbol{N}=\mathbf{1 8})\end{array}$ & $\begin{array}{l}\text { Cannabis users } \\
(\mathbf{N = 1 8})\end{array}$ \\
\hline Age (years) & $17.2 \pm 0.82$ & $17.7 \pm 0.94$ \\
Sex (M) & $+6 \quad 12$ & $+2 \quad 116$ \\
Handedness (M) & 18 Right handed & 18 Right handed \\
Age at first use (years) & - & $14.86 \pm 0.31$ \\
Age at regular use (years) & - & $15.72 \pm 0.20$ \\
Average frequency/ week & - & $9.13 \pm 1.96$ \\
Estimated lifetime uses & - & $1351.94 \pm 322.54$ \\
THCCOOH (ng) & - & $429.16 \pm 84.63$ \\
Family history of substance abuse & - & $50 \%$ \\
History of other substance abuse & - & $11 \% *$ \\
Current other substance abuse & - & - \\
History of tobacco use & - & $22 \%$ \\
Current tobacco use & - & $22 \%$ \\
History of psychiatric diagnosis & - & $5 \% *$ \\
Current psychiatric diagnosis & - & - \\
HAM-A & $1.27 \pm 0.45$ & $1.89 \pm 0.62$ \\
HAM-D & $0.78 \pm 0.33$ & $2.0 \pm 0.71$ \\
& &
\end{tabular}

Mean and SE for demographic variables, cannabis use dimensions, and HAM-A/ HAM-D scores. Additionally, percentage of cannabis abusing and healthy control subjects with positive family history of substance abuse, history or current other substance abuse/tobacco use, or psychiatric diagnoses are reported. Estimates of lifetime cannabis use were calculated by summing the number of weeks since the age of reported first regular use until the week of the study visit and multiplying that value by the reported average frequency (episodes) of use per week.

HAM-A, Hamilton anxiety rating scale; HAM-D, Hamilton depression rating scale.

* Both identified subjects had a history of alcohol use.

**The identified subject had a history of major depression. used. The surface-based analysis involved registration with the Talairach and Tournoux atlas, intensity normalization, and skull stripping, followed by white matter labeling (Dale et al., 1999). Cortical white matter surfaces were used in a deformation procedure that assigns gray and white matter borders by following intensity gradients to the position at which the maximum shift in intensity designated the transition to the other tissue class (Fischl and Dale, 2000). An automated registration procedure was used to label each voxel in an MRI volume based on probabilities estimated from a manually labeled training set (Fischl et al., 2002). Standard FreeSurfer predefined region of interest (ROI) labels for moPFC and lateral orbital prefrontal cortex (loPFC) were used in the final statistical analysis. Robust correlations between the predefined ROIs and manual tracing procedures have been demonstrated in both of these regions (Desikan et al., 2006). After the analyses were completed, volume data for ROIs in moPFC and loPFC were extracted from the imaging analysis environment and submitted to statistical testing.

\section{RESULTS \\ DATA REDUCTION AND STATISTICAL ANALYSIS}

Initially, values for brain volumes were obtained by taking the ratio of regional (ROIs) to total segmented brain volume for each subject and multiplying the obtained quotient by 100 , hereafter referred to as volume (see Table 2 ).

Total segmented brain volumes included all brain regions identified in FreeSurfer aseg.mgz. This set of measures includes the ventricles, but excludes the dura. An estimate of lifetime cannabis use was also calculated by summing the number of weeks since the age of reported first regular use multiplied by the reported average frequency (episodes) of use per week. Following transformations, all data were analyzed using SPSS 17 for Mac.

Previous research indicates that early initiation of cannabis use is associated with reduced frontal cortical gray matter (Wilson et al., 2000) and that right moPFC volume is negatively correlated with non-planning impulsivity (Matsuo et al., 2009). Therefore,

\begin{tabular}{lllll}
\hline Table 2 | Regions of interest. & & & \\
\hline & $\begin{array}{l}\text { Healthy controls } \\
(\boldsymbol{n}=\mathbf{1 8})\end{array}$ & $\begin{array}{l}\text { Cannabis } \\
\text { users }(\boldsymbol{n}=\mathbf{1 8})\end{array}$ & $\boldsymbol{p}$ & $\boldsymbol{d}$ \\
& Mean \pm SE & Mean \pm SE & & \\
& $0.644 \pm 0.014$ & $0.644 \pm 0.013$ & 0.97 & 0.01 \\
& $0.656 \pm 0.015$ & $0.653 \pm 0.013$ & 0.87 & 0.05 \\
L loPFC & $1.301 \pm 0.027$ & $1.297 \pm 0.024$ & 0.91 & 0.09 \\
R loPFC & $0.370 \pm 0.009$ & $0.387 \pm 0.022$ & 0.92 & 0.04 \\
Total loPFC & $0.402 \pm 0.008$ & $0.369 \pm 0.013$ & $0.01^{*}$ & 0.92 \\
L moPFC & $0.772 \pm 0.015$ & $0.737 \pm 0.017$ & 0.13 & 0.51 \\
R moPFC & $2.074 \pm 0.038$ & $2.035 \pm 0.035$ & 0.54 & 0.25 \\
Total moPFC & & & & \\
Total orbital PFC & &
\end{tabular}

Mean, SE, p-values, and Cohen's $d$ for adjusted volumes in $\mathrm{mm}^{3}$ for regional and total ROls. Values for brain volumes were obtained by taking the ratio of regional to total segmented brain volume for each subject and multiplying the obtained quotient by 100 .

$R O I$, region of interest; $L$, left and $R$, right; loPFC, lateral orbital prefrontal cortex; moPFC, medial orbital prefrontal cortex.

*Indicates significant difference between groups. 
we tested the hypothesis that cannabis abusing adolescents have altered moPFC volume and report increased impulsivity compared to healthy controls. Bonferroni adjusted independent-samples $t$-tests were conducted for moPFC volumes and a significant difference between groups for right moPFC volume $t(34)=2.74, p=0.01, d=0.92, \mathrm{CI}_{0.95}=0.21,1.59$, but not left moPFC volume $t(34)=0.1, p=0.92$, or total moPFC volume $t(34)=1.51, p=0.13$ was demonstrated. CA adolescents showed reduced volume in right moPFC compared to controls (Figures 1 and 2). Bonferroni adjusted independent-samples $t$-tests were conducted for impulsivity measures (non-planning, attention, motor, and total) and a significant difference between groups for the BIS non-planning subscale was demonstrated $t(34)=-2.66$, $p=0.01, d=0.89, \mathrm{CI}_{0.95}=0.23,1.55$, with cannabis abusing adolescents showing increased non-planning impulsivity compared to HCs. However, there were no significant differences between

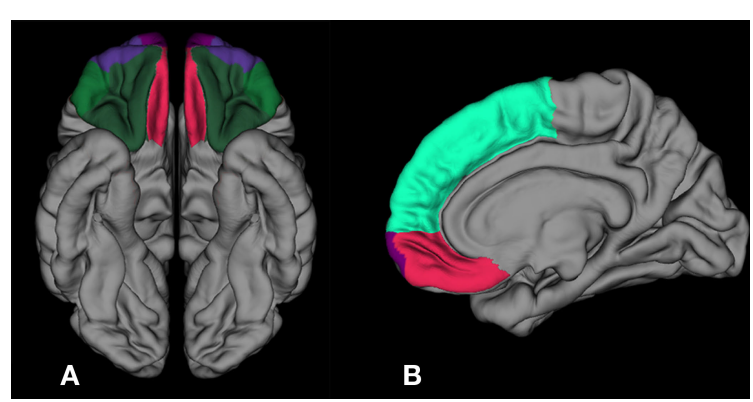

FIGURE 1 | Inferior and sagittal 3-D representations with ROls and reference regions employed in FreeSurfer including: (A) moPFC in red, IoPFC in dark green, and frontal pole in purple and (B) medial view showing superior PFC in light green, moPFC in red, and frontal pole in purple. ROI, region of interest; $\mathrm{PFC}$, prefrontal cortex; loPFC, lateral orbital prefrontal cortex; moPFC, medial orbital prefrontal cortex.

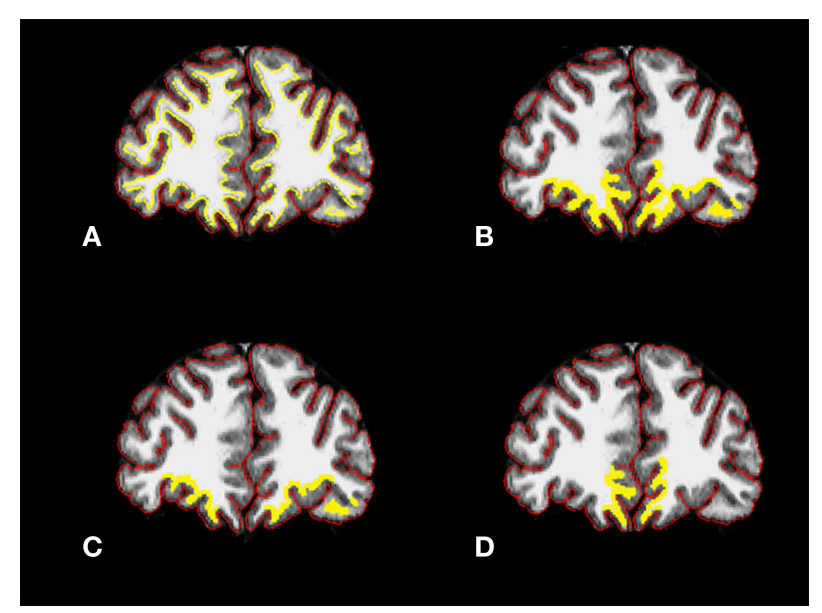

FIGURE 2 | Coronal sections from CA subject with (A) cortical (red) and white matter (yellow) surfaces outlined, (B) ROI in entire orbital prefrontal cortical region, (C) ROI in loPFC, and (D) ROI in moPFC. CA, cannabis abusing; $\mathrm{ROI}$, region of interest; loPFC, lateral orbital prefrontal cortex; moPFC, medial orbital prefrontal cortex. Yellow ROI line was thickened for visibility in (B-D). groups for any other measures of impulsivity including attention $t(34)=-1.21, p=0.23$, motor $t(34)=-0.13, p=0.89$, or total impulsivity $t(34)=-1.55, p=0.13$.

Exploratory analysis revealed no significant differences between groups for regional lateral orbitofrontal volumes or total orbital PFC volume: right loPFC, $p=0.87$; left loPFC volume, $p=0.97$; total loPFC volume, $p=0.91$; total orbital PFC volume, $p=0.45$. A separate exploratory analysis using all orbital prefrontal regions (see Table 2) did, however, show a modest positive relationship between age of first use and left moPFC volume $r(18)=0.57, p=0.01$ as well as between age of first use and total moPFC volume $r(18)=0.49$, $p=0.03$ (Figure 3).

\section{DISCUSSION}

The results of the present investigation indicate that, compared to healthy controls, cannabis abusing adolescents have decreased right moPFC volume and also report increased impulsivity related to decreased future orientation as indexed by the BIS non-planning subscale. While there were no significant correlations between right moPFC volume and several measures of cannabis use, total moPFC volume was positively associated with age of first use.

Damage to the medial prefrontal cortex has been shown to impair decision making using the Iowa gambling task (IGT) (Bechara et al., 1994, 1999) and cannabis users also show diminished choice optimization and decreased functional activation of orbital prefrontal regions during performance of the IGT (Bolla et al., 2005; Verdejo-Garcia et al., 2007). Suboptimal performance on the IGT in healthy college-aged students has been associated with higher scores on the BIS (Sweitzer et al., 2008), further suggesting

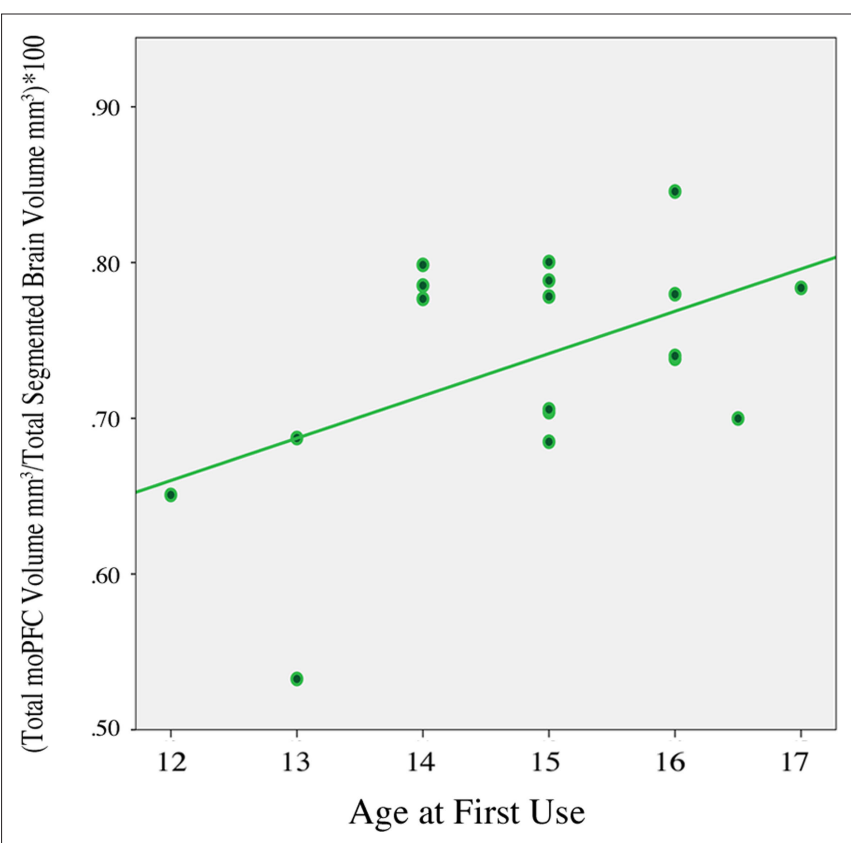

FIGURE 3 | Correlation between total moPFC volume and age of first use in adolescent cannabis users, $\boldsymbol{r}(\mathbf{1 8}) \mathbf{0 . 4 9}, \boldsymbol{p}=\mathbf{0 . 0 3}$. Two scores overlap in scatter plot, $(N=18)$. moPFC, medial orbital prefrontal cortex. 
that cannabis use is related to altered orbital prefrontal function, impaired decision making, and possibly self-reported impulsivity. Although, our data did not indicate a correlation between total moPFC volume and impulsivity, our findings are in line with these studies since cannabis abusing participants in the current study did show altered moPFC volume and increased non-planning impulsivity. Our results thus support the proposal that cannabis abuse is related to dysregulated moPFC functioning and the ability to reflect on future outcomes. Consistent with these findings, previous research using a sample of healthy adolescent boys showed reduced right moPFC volume to be associated with higher levels of observed impulsivity (Boes et al., 2009). Matsuo et al. (2009) also found the BIS non-planning subscale to be negatively correlated with right orbital prefrontal volume in a community sample of healthy adults.

Shortsightedness during adolescence may be driven by an enhanced motivation to seek novelty and cannabis abuse may be related to this drive. For example, increased self-reported novelty seeking has been demonstrated in cannabis users (Hale et al., 2003) and increased frequency of use has been associated with heightened sensation seeking (Eisenman et al., 1980). Interestingly, there is evidence to suggest a potential relationship among novelty seeking, adolescent cannabis abuse, and basal density of $\mathrm{CB}_{1}$ receptors. For example, a positron emission tomography (PET) study in a large sample of healthy adult volunteers demonstrated that $\mathrm{CB}_{1}$ receptor availability was inversely correlated with selfreported novelty seeking (Van Laere et al., 2009). $\mathrm{CB}_{1}$ receptor density is thought to undergo change in the prefrontal cortex and nucleus accumbens during adolescence (Ellgren et al., 2008) and treatment with the synthetic cannabinoid HU210 has been shown to reduce $\mathrm{CB}_{1}$ receptor density in cortical and subcortical brain regions in a preclinical model of adolescent substance abuse (Dalton and Zavitsanou, 2010). A separate line of research has suggested that cannabis use may adversely impact reward systems or drug-related cue salience. For example, a study examining the effect of a reward task on mood in adult cannabis users and non-users found that monetary reward had a positive impact on mood in non-users participating in a spatial recognition task, but had no such effect in cannabis users (Martin-Soelch et al., 2009). Moreover, the prefrontal-accumbens circuit is normally involved in reward processing related to goal-directed behavior and cellular adaptations resulting from repeated drug exposure are thought to critically modify this circuit so that it becomes biased toward drug seeking (Kalivas and Volkow, 2005). Consistent with this notion, a study that investigated functional brain activation in response to cannabis cues compared to neutral cues, in briefly abstinent adult users, showed activity in orbitofrontal cortex and nucleus accumbens positively correlated with the marijuana problem scale (Filbey et al., 2009), suggesting that increased self-reported negative family, social, legal, and occupational consequences related to cannabis abuse are associated with increased activation within the prefrontal-accumbens circuit. Collectively, these studies suggest that adolescent cannabis use may be related to altered reward seeking and drug-related reward salience as well as neural sensitization, attenuation of normal cannabinoid system development, and alterations in brain regions associated with reward and decision making.
Delayed maturation of frontal systems during adolescence is also thought to contribute to impaired decision making and shortsightedness (Galvan et al., 2006; Ernst et al., 2008; Fareri et al., 2008; Steinberg et al., 2009) and protracted development of the prefrontal cortex may be importantly related to emotional dysregulation during this developmental period (Yurgelun-Todd, 2007). For example, Killgore and Yurgelun-Todd (2007) used fMRI to test the neural correlates of emotional reactivity in adolescents using an experimental paradigm in which fearful faces were shown. They further characterized emotional intelligence in the same group using the Bar-On emotional quotient inventory (EQ-i). The results of the study suggested that adolescent EQ was associated with functional activation within several regions implicated in the integration of cognitive and emotional information and an inverse relationship between EQ-i score and activity within the ventromedial prefrontal cortex during viewing of fearful faces was specifically observed. These results suggest that increased activation in the ventromedial prefrontal cortex is associated with poorer EQ-scores, consistent with reduced neural efficiency in processing emotional information in low EQ adolescents. Given that the same region is also implicated in cannabis use in the present investigation, it is quite possible that some adolescent cannabis users may have diminished emotional intelligence. Insensitivity to future outcomes may be a specific impairment within this general capacity. Thus, some adolescents may be especially vulnerable to initiating cannabis use during this time due to delayed emotional development and a corresponding reduced capacity to use emotional information to guide decisions. However, cannabis use may impact both normal emotional and neural development and lead to long-term developmental slowing (Figure 4). Consistent with this idea, the transition from drug initiation to dependence is thought to results in potent neuroplastic changes and structural reorganization (Koob and Le Moal, 2005; Koob and Volkow, 2010), which may in turn impact the capacity for life experiences to induce plasticity and provide the cognitive and behavioral advantages associated with normal maturation (Robinson and Kolb, 2004; Koob and Volkow, 2010).

While the present results may have implications for understanding adolescent decision making and neural development with respect to cannabis use, possible inferences should be cautiously considered within the context of the limitations of this study. For

Future
Orientation
ElGURE 4 | Possible relational
emotional development, substance abuse, and prospective thinking in
decision making. Multiple entry points within the model (e.g., prefrontal
development or cannabis abuse) suggest that a window of vulnerability may
occur during normal neurodevelopment, emotional development and
decision making, and may increase risk for cannabis abuse. Conversely,
normal neurodevelopment may be altered by cannabis abuse, disrupt
emotional development and decision-making capacities, and increase risk for
compulsive drug use.


example, the sample size used in this study was modest and groups were not gender matched, which may prevent generalization of these findings. Specifically, a differential impact of cannabis use on a measure of entire prefrontal cortical volume (including gray and white matter) for abstinent male and female users has been observed (Medina et al., 2009).

A further limiting factor in this study is the manner in which impulsivity was measured. The BIS is a self-report instrument and thus may be susceptible to over or underestimation based on socially desirable responding or inaccurate self-perception (Meda et al., 2009). Similarly, the relationship between laboratory and selfreport measures of impulsivity and their respective association with frontal morphology for both healthy and substance abusing populations is yet unclear (Matsuo et al., 2009). There is some evidence to suggest that laboratory and self-report measures are unrelated in adults and adolescents (Reynolds et al., 2008). For example, Bjork et al. (2009) did not observe any associations between frontal gray matter volumes and scores on the BIS in a healthy adult population. However, they used a delay discounting task to demonstrate that increased preference for immediate over delayed rewards is associated with decreased gray matter volume in dorsolateral and inferolateral prefrontal cortex. However, functional activation of medial prefrontal cortex during delay discounting has also been linked to the subjective value of rewards (Kable and Glimcher, 2007), suggesting a central role for this region in evaluating immediate versus remote choice outcomes.

An additional consideration in the present study is that cannabis abusing participants were instructed to continue using as usual until study day. It is possible that recent use may have influenced responding on the BIS. However, there was no correlation between tetrahydrocannabinol metabolite values and BIS scores, suggesting

\section{REFERENCES}

Barratt, E. S. (1994). "Impulsiveness and aggression," in Violence and Mental Disorder: Developments in Risk Assessment, ed. J. M. H. J. Steadman (Chicago: University of Chicago Press), 61-79.

Bechara, A. (2005). Decision making, impulse control and loss of willpower to resist drugs: a neurocognitive perspective. Nat. Neurosci. 8 , 1458-1463.

Bechara, A., Damasio, A. R., Damasio, H., and Anderson, S. W. (1994). Insensitivity to future consequences following damage to human prefrontal cortex. Cognition 50, 7-15.

Bechara, A., Damasio, H., Damasio, A. R., and Lee, G. P. (1999). Different contributions of the human amygdala and ventromedial prefrontal cortex to decision-making. J. Neurosci. 19, 5473-5481.

Bechara, A., Tranel, D., and Damasio, H. (2000). Characterization of the decision-making deficit of patients with ventromedial prefrontal cortex lesions. Brain 123(Pt 11), 2189-2202.
Berlin, H. A., Rolls, E. T., and Kischka, U. (2004). Impulsivity, time perception, emotion and reinforcement sensitivity in patients with orbitofrontal cortex lesions. Brain 127(Pt 5), 1108-1126.

Bjork, J. M., Momenan, R., and Hommer, D.W. (2009). Delay discounting correlates with proportional lateral frontal cortex volumes. Biol. Psychiatry 65, 710-713.

Boes, A. D., Bechara, A., Tranel, D., Anderson, S. W., Richman, L., and Nopoulos, P. (2009). Right ventromedial prefrontal cortex: a neuroanatomical correlate of impulse control in boys. Soc. Cogn. Affect. Neurosci. 4, 1-9.

Bolla, K. I., Eldreth, D. A., Matochik, J. A., and Cadet, J. L. (2005). Neural substrates of faulty decision-making in abstinent marijuana users. Neuroimage 26, 480-492.

Chen, O'Brien, S. M., and Anthony, J. C. (2005). Who becomes cannabis dependent soon after onset of use? United States: 2000-2001. Drug Alcohol Depend. 79, 11-22. Epidemiological evidence from the

that active levels did not influence BIS responding. Lastly, this study employed a cross sectional design and it cannot be determined whether cannabis use produces alterations in frontal morphology and impulsivity or whether these differences existed prior to and contributed to initiation.

Of 2.7 million people 12 years or older in the U.S. to initiate drug use in 2007, cannabis was the number one drug of choice, constituting 56.2\% of initiates (SAMHSA, 2008). Cannabis is readily available to adolescents and nearly $32 \%$ of 12 th graders use cannabis annually (Johnston et al., 2008). It has been proposed that early initiation of cannabis use may increase the risk for cannabis dependence (Chen et al., 2005) and that heavy cannabis use may also have a negative neurocognitive impact (Pope et al., 2001; Schweinsburg et al., 2008). Given that the transition from childhood to adulthood is a significant time of neural and emotional change, the impact of cannabis use may be a critical determinant in long-term outcomes for some individuals with respect to substance abuse disorder and general trajectory. The present results suggest that adolescent cannabis users have reduced right moPFC volume and that age of first use and volume reductions in this region are also related. Adolescent cannabis users also showed increased levels of non-planning impulsivity, suggesting a possible source of impaired decision making. Going forward, characterizing neural, decision making, and behavioral dimensions of adolescent cannabis abuse will be critical for determining biomarkers of risk, in addition to being essential for optimizing intervention and treatment strategies (Churchwell and Yurgelun-Todd, in press).

\section{ACKNOWLEDGMENT}

Research supported by NIH grant 1R01 DA020269-01 to Deborah A. Yurgelun-Todd.

Churchwell, J. C., and Yurgelun-Todd, D. A. (in press). "Neuroimaging, adolescence and riskybehavior," in Inhibitory Control and Drug Abuse Prevention: From Research to Translation, eds M.T. Bardo, D. H. Fishbein, and R. Milich.

Crews, F., He, J., and Hodge, C. (2007). Adolescent cortical development: a critical period of vulnerability for addiction. Pharmacol.Biochem. Behav. 86, 189-199.

Dale, A. M., Fischl, B., and Sereno, M. I. (1999). Cortical surface-based analysis. I. Segmentation and surface reconstruction. Neuroimage 9 , 179-194.

Dalton, V. S., and Zavitsanou, K. (2010). Cannabinoid effects on $\mathrm{CB} 1$ receptor density in the adolescent brain: an autoradiographic study using the synthetic cannabinoid HU210. Synapse 64, 845-854.

Desikan, R. S., Segonne, F., Fischl, B., Quinn, B. T., Dickerson, B.C., Blacker, D., Buckner, R. L., Dale,A.M., Maquire, R. P., Hyman, B. T., Albert, M. S., and Killiany, R. J. (2006). An automated labeling system for subdividing the human cerebral cortex on MRI scans into gyral based regions of interest. Neuroimage 31, 968-980.

Egerton, A., Allison, C., Brett, R. R., and Pratt, J. A. (2006). Cannabinoids and prefrontal cortical function: insights from preclinical studies. Neurosci. Biobehav. Rev. 30, 680-695.

Eisenman, R., Grossman, J. C., and Goldstein, R. (1980). Undergraduate marijuana use as related to internal sensation novelty seeking and openness to experience. J. Clin. Psychol. 36, 1013-1019.

Ellgren, M., Artmann, A., Tkalych, O., Gupta, A., Hansen, H. S.,Hansen, S. H., Devi, L. A., and Hurd, Y. L. (2008). Dynamic changes of the endogenous cannabinoid and opioid mesocorticolimbic systems during adolescence: THC effects. Eur. Neuropsychopharmacol. 18, 826-834.

Ellgren, M., Spano, S. M., and Hurd, Y. L. (2007). Adolescent cannabis exposure alters opiate intake and opioid limbic neuronal populations in adult rats. Neuropsychopharmacology 32, 607-615.

Ernst, M., and Fudge, J. L. (2009). A developmental neurobiological model of 
motivated behavior: anatomy, connectivity and ontogeny of the triadic nodes. Neurosci. Biobehav. Rev. 33, 367-382.

Ernst, M., Romeo, R. D., and Andersen, S. L. (2008). Neurobiology of the development of motivated behaviors in adolescence: a window into a neural systems model. Pharmacol. Biochem. Behav. 93, 199-211.

Fareri, D. S., Martin, L. N., and Delgado, M. R. (2008). Reward-related processing in the human brain: developmental considerations. Dev. Psychopathol. 20, 1191-1211.

Filbey, F. M., Schacht, J. P., Myers, U. S., Chavez, R. S., and Hutchison, K. E. (2009). Marijuana craving in the brain. Proc. Natl. Acad. Sci. U.S.A. 106, 13016-13021.

Fischl, B., and Dale, A. M. (2000). Measuring the thickness of the human cerebral cortex from magnetic resonance images. Proc. Natl. Acad. Sci. U.S.A. 97, 11050-11055.

Fischl, B., Salat, D. H., Busa, E., Albert, M., Dieterich, M., Haselgrove, C., van der Kouwe, A., Killiany, R., Kennedy, D., Klaveness, S., Montillo, A., Markis, N., Rosen, B., and Dale, A. M. (2002). Whole brain segmentation: automated labeling of neuroanatomical structures in the human brain. Neuron 33, 341-355.

Fuster, J. M. (2002). Frontal lobe and cognitive development. J. Neurocytol. 31, 373-385.

Galvan, A., Hare, T. A., Parra, C. E., Penn, J., Voss, H., Glover, G., and Casey, B. (2006). Earlier development of the accumbens relative to orbitofrontal cortex might underlie risk-taking behavior in adolescents. J. Neurosci. 26, 6885-6892.

Hale, R. L., Whiteman, S., Muehl, K., and Faynberg, E. (2003). Tridimensional personality traits of college student marijuana users. Psychol. Rep. 92, 661-666.

Hamilton, A. (1969). Diagnosis and rating of anxiety. Br. J. Psychiatry Special Issue 3, 76-79.

Hamilton, M. (1960). A rating scale for depression. J. Neurol. Neurosurg. Psychiatr. 23, 56-62.

Higuera-Matas, A., Soto-Montenegro, M. L., del Olmo, N., Miguens, M., Torres, I., Vaquero, J. J., Sanchez, J., Garcia-Lecumberri,C., Desco, M., and Ambrosio, E. (2008). Augmented acquisition of cocaine self-administration and altered brain glucose metabolism in adult female but not male rats exposed to a cannabinoid agonist during adolescence. Neuropsychopharmacology 33 , 806-813.

Jacobus, J., Bava, S., Cohen-Zion, M., Mahmood, O., and Tapert, S. F. (2009).
Functional consequences of marijuana use in adolescents. Pharmacol. Biochem. Behav. 92, 559-565.

Johnston, L. D., O’Malley, P.M., Bachman, J. G., and Schulenberg, J. E. (2008). Monitoring the Future National Results on Adolescent Drug Use. Overview of Key Findings, 2007 (NIH Publication No.08-6418). Bethesda, MD: National Institute on Drug Abuse.

Kable, J. W., and Glimcher, P. W. (2007). The neural correlates of subjective value during intertemporal choice. Nat. Neurosci. 10, 1625-1633.

Kalivas, P. W., and Volkow, N. D. (2005). The neural basis of addiction: a pathology of motivation and choice. Am. J. Psychiatry 162, 1403-1413.

Kanayama, G., Rogowska, J., Pope, H. G., Gruber, S. A., and Yurgelun-Todd, D. A. (2004). Spatial working memory in heavy cannabis users: a functional magnetic resonance imaging study. Psychopharmacology (Berl), 176, 239-247.

Killgore, W. D., and Yurgelun-Todd, D. A. (2007). Neural correlates of emotional intelligence in adolescent children. Cogn. Affect. Behav. Neurosci. 7, 140-151.

Kolb, B., Gorny, G., Limebeer, C. L., and Parker,L.A. (2006). Chronic treatment with Delta-9-tetrahydrocannabinol alters the structure of neurons in the nucleus accumbens shell and medial prefrontal cortex of rats. Synapse 60, 429-436.

Koob, G. F., and Le Moal, M. (2005). Plasticity of reward neurocircuitry and the "dark side" of drug addiction. Nat. Neurosci. 8, 1442-1444.

Koob, G. F., and Volkow, N. D. (2010). Neurocircuitry of addiction. Neuropsychopharmacology 35, 217-238.

Luna, B., and Sweeney, J. A. (2004). The emergence of collaborative brain function: FMRI studies of the development of response inhibition. Ann. N. Y.Acad. Sci. 1021, 296-309.

Martin-Soelch, C., Kobel, M., Stoecklin, M., Michael, T., Weber, S., Krebs, B., and Opwis, K. (2009). Reduced response to reward in smokers and cannabis users. Neuropsychobiology 60, 94-103.

Matsuo, K., Nicoletti, M., Nemoto, K., Hatch, J. P., Peluso, M. A., Nery, F. G., and Soares, J. C. (2009). A voxel-based morphometry study of frontal gray matter correlates of impulsivity. Hum. Brain Mapp. 30, 1188-1195.

McNamee, R. L., Dunfee, K. L., Luna, B., Clark, D. B., Eddy, W. F., and Tarter, R. E. (2008). Brain activation, response inhibition, and increased risk for substance use disorder. Alcohol. Clin. Exp. Res. 32, 405-413.
Meda, S. A., Stevens, M. C., Potenza, M. N., Pittman, B., Gueorguieva, R., Andrews, M., Thomas, A. D., Muska, C., Hylton, J. L., and Pearlson, G. D. (2009). Investigating the behavioral and self-report constructs of impulsivity domains using principal component analysis. Behav. Pharmacol. 20, 390-399.

Medina, K. L., Hanson, K. L., Schweinsburg, A. D., Cohen-Zion, M., Nagel, B. J., and Tapert, S. F. (2007). Neuropsychological functioning in adolescent marijuana users: subtle deficits detectable after a month of abstinence. J. Int. Neuropsychol. Soc. 13, 807-820.

Medina, K. L., McQueeny, T., Nagel, B. J., Hanson, K. L., Yang, T. T., and Tapert, S. F. (2009). Prefrontal cortex morphometry in abstinent adolescent marijuana users: subtle gender effects. Addict. Biol. 14, 457-468.

Moeller, F. G., Barratt, E. S., Dougherty, D. M., Schmitz, J. M., and Swann, A. C. (2001). Psychiatric aspects of impulsivity. Am. J. Psychiatry 158, 1783-1793.

Patton, J. H., Stanford, M. S., and Barratt, E. S. (1995). Factor structure of the Barratt impulsiveness scale. J. Clin. Psychol. 51, 768-774.

Pope, H. G. Jr., Gruber, A. J., Hudson, J. I., Cohane, G., Huestis, M. A., and Yurgelun-Todd, D. (2003). Earlyonset cannabis use and cognitive deficits: what is the nature of the association? Drug Alcohol Depend. 69, 303-310.

Pope, H. G. Jr., Gruber, A. J., Hudson, J. I., Huestis, M. A., and YurgelunTodd, D. (2001). Neuropsychological performance in long-term cannabis users. Arch. Gen. Psychiatry 58, 909-915.

Reynolds, B., Penfold, R. B., and Patak, M. (2008). Dimensions of impulsive behavior in adolescents: laboratory behavioral assessments. Exp. Clin. Psychopharmacol. 16, 124-131.

Robinson, T. E., and Berridge, K. C. (1993). The neural basis of drug craving: an incentive-sensitization theory of addiction. Brain Res. Brain Res. Rev. 18, 247-291.

Robinson, T. E., and Kolb, B. (2004). Structural plasticity associated with exposure to drugs of abuse. Neuropharmacology 47(Suppl. 1), 33-46.

Rolls, E. T., and Grabenhorst, F. (2008). The orbitofrontal cortex and beyond: from affect to decision-making. Prog. Neurobiol. 86, 216-244.

Schepis, T. S., Adinoff, B., and Rao, U. (2008). Neurobiological processes in adolescent addictive disorders. Am. J. Addict. 17, 6-23.
Schweinsburg, A. D., Brown, S. A., and Tapert, S. F. (2008). The influence of marijuana use on neurocognitive functioning in adolescents. Curr. Drug Abuse Rev. 1, 99-111.

Schweinsburg, A. D., Nagel, B. J., Schweinsburg, B. C., Park, A., Theilmann, R. J., and Tapert, S. F. (2008). Abstinent adolescent marijuana users show altered fMRI response during spatial working memory. Psychiatry Res. 163, 40-51.

Somerville, L. H., and Casey, B. (2010). Developmental neurobiology of cognitive control and motivational systems. Curr. Opin. Neurobiol. 20, 236-241.

Spear, L. P. (2000). The adolescent brain and age-related behavioral manifestations. Neurosci. Biobehav. Rev. 24, 417-463.

Steinberg, L., Graham, S., O’Brien, L., Woolard, J., Cauffman, E., and Banich, M. (2009). Age differences in future orientation and delay discounting. Child Dev. 80, 28-44.

Strauss, E., Sherman, E. M. S., and Spreen, O. (2006). A Compendium of Neuropsychological Tests: Administration, Norms, and Commentary, 3rd Edn. Oxford, New York: Oxford University Press.

Substance Abuse and Mental Health Services Administration (SAMHSA). (2008). Results from the 2007 National Survey on Drug Use and Health: National Findings. Rockville, MD: SAMHSA.

Sweitzer, M. M., Allen, P. A., and Kaut, K. P. (2008). Relation of individual differences in impulsivity to nonclinical emotional decision making. J. Int Neuropsychol. Soc. 14, 878-882.

United Nations (UN). (2008). "United Nations office on drugs and crime," in: World Drug Report 2008. Vienna: United Nations Publications.

Van Laere, K., Goffin, K., Bormans, G., Casteels, C., Mortelmans, L., de Hoon, J., Grachev, I., Vandenbulcke, M., and Pieters, G. (2009). Relationship of type 1 cannabinoid receptor availability in the human brain to novelty-seeking temperament. Arch. Gen. Psychiatry 66, 196-204.

Verdejo-Garcia, A., Benbrook, A., Funderburk, F., David, P., Cadet, J. L., and Bolla, K. I. (2007). The differential relationship between cocaine use and marijuana use on decision-making performance over repeat testing with the Iowa Gambling Task. Drug Alcohol Depend. 90, 2-11.

Wilson, W., Mathew, R., Turkington, T., Hawk, T., Coleman, R.E., and Provenzale, J. (2000). Brain morphological changes and early marijuana use: a magnetic resonance and positron emission tomography study. J Addict. Dis. 19, 1-22. 
Yurgelun-Todd. (2007). Emotional and cognitive changes during adolescence. Curr. Opin. Neurobiol. 17, 251-257.

Conflict of Interest Statement: The authors declare that the research was conducted in the absence of any com- mercial or financial relationships that could be construed as a potential conflict of interest.

Received: 27 August 2010; paper pending published: 28 September 2010; accepted: 29 November 2010; published online: 14 December 2010.
Citation: Churchwell JC, Lopez-Larson M and Yurgelun-Todd DA (2010) Altered frontal cortical volume and decision making in adolescent cannabis users. Front. Psychology 1:225. doi: 10.3389/fpsyg.2010.00225

This article was submitted to Frontiers in Psychopathology, a specialty of Frontiers in Psychology.
Copyright () 2010 Churchwell, LopezLarson and Yurgelun-Todd. This is an open-access article subject to an exclusive license agreement between the authors and the Frontiers Research Foundation, which permits unrestricted use, distribution, and reproduction in any medium, provided the original authors and source are credited. 